\title{
Editorial
}

\section{ELIMINATION OF LEPROSY AS A PUBLIC HEALTH PROBLEM}

The Forty-fourth World Health Assembly, which met in May 1991, adopted a resolution on the elimination of leprosy as a public health problem by the year 2000, and defined this elimination as attaining a level of prevalence below 1 case per 10,000 population. This landmark resolution declared WHO's commitment to attain global elimination after the Assembly noted the significant progress made with multidrug therapy (MDT) and the consequent reduction in disease prevalence, as well as the substantial support from nongovernmental organizations (NGOs), and the increased priority accorded to leprosy control by several of its Member States. The resolution, among others, further urged Member States to increase their political commitment for leprosy in order to reach the elimination goal, and requested the Director-General of WHO to strengthen technical support to Member States and to continue to mobilize and coordinate resources from NGOs and others in order to achieve the goal.

The World Health Assembly, by establishing a target for the year 2000, has drawn attention to the effectiveness of the available treatment technology, the need for leprosyendemic countries and donor agencies to cease to regard leprosy as a permanent problem but to redouble their efforts toward leprosy control, and the need to accept leprosy as simply another health problem with a clear solution.

It is clear that MDT offers an opportunity not available before. Experience in several countries in the past 8 years or so has demonstrated convincingly that it is possible to reduce the prevalence of registered cases up to 10 -fold within a period of 5 years, provided MDT is applied to at least $80-90 \%$ of registered cases. However, what is not always clear in any given situation is the extent to which the number of registered cases reflects the true situation in terms of existing cases, including the unregistered, 'hidden' cases. While this problem of unregistered cases is partly due to nonrecognition of the disease by the patient and others around him, particularly of early disease, a significant part of the problem is also due to the concealment of the known disease because of its associated social stigma, which can be quite severe, as observed in many communities. Well-organized leprosy control programmes have tried to address both aspects of this problem through health education aimed at creating awareness towards recognition of early disease and breaking down the social barriers that hinder patients self-reporting to the health services. However, the introduction of MDT, in itself, appears to have increased self-reporting in many programmes as a result of the perception by the patient and the community that it is a highly effective treatment, leading to definite cure. With this encouraging trend, it is hoped that the gap between the registered and estimated cases will steadily decrease over a period of time and that the residual unregistered cases will consist of mainly early minimal 
and mostly self-healing paucibacillary cases with a very low potential for transmission of infection.

Another consequence of the early self-reporting of patients, together with prompt administration of MDT, would be that after a period of time the prevalent cases would consist chiefly of incident cases, thus reflecting the more recent changes taking place with regard to the epidemiological situation. In this connection, the more sensitive indicators to monitoring for epidemiological changes will be changes in the incidence of leprosy in the younger age groups even though the disease might occur in all ages. This is because, while disease incidence in younger ages, as in tuberculosis, is likely to reflect recent infections, disease in older ages, particularly in persons over 30 or 40 years, is likely to be due primarily to endogenous reactivation of infections acquired in childhood. Thus, with the effective application of MDT in all patients and with an overall declining trend, it is unlikely that the continued low-level incidence of leprosy in older individuals would indicate any recent transmission of infection with Mycobacterium leprae, although such low-level disease incidence will make it impossible to envisage the total eradication of leprosy, even in a time span of 10 to 20 years. Hence the use of the term 'elimination of leprosy as a public health problem', clearly defining elimination as attaining a prevalence level below 1 case per 10,000 population, appears to be quite appropriate.

The elimination of leprosy as a public health problem aims essentially at the elimination of infection through MDT, thus preventing transmission. However, it should be recognized that leprosy is dreaded not because of its infection but because of the deformities it produces with their serious physical, social and psychological consequences. While MDT, through early cure, will contribute substantially to the prevention of deformities and thus will have a long-term impact, it will have very little impact on those patients who are already deformed.

It is estimated that there are about two to three million individuals in the world who are disabled physically and socially as a result of either past or present leprosy. A large proportion of these disabled persons will survive the turn of the century. In addition, a significant proportion of new cases occurring in the 1990s, and even some old patients from preceding decades, will develop deformities resulting from late diagnosis and insufficient care. Although such individuals could be cured, from the microbial point of - view, through MDT and thus eliminated as cases for the purpose of public health control, the problem of disability will persist and so warrant continued care. Thus, even if elimination of leprosy as a public health problem is attained, it is clear that the problem of disability will persist well into the next century. The only way this could be mitigated is by substantial improvements in the current technologies for prevention and management of nerve damage and deformities and their application in the field. In terms of social disability, MDT appears to have a positive influence in reducing stigma against the disease. Patients, community and health workers alike now have a much better perception than ever before of the curability of the disease.

What then are the implications of the elimination strategy? First, it is obvious that elimination is not total eradication of the disease, and that we are willing to accept a small residual problem, this being defined as 1 case per 10,000 population in the hope that when such very low levels are reached the transmission of infection will be so minimal that the disease in the community will die out in the course of time. In this connection, a point often raised is what about the possibility of a resurgence of the disease af ter attaining low levels of prevalence? Historically, there is no evidence of resurgence of the disease in areas 
where it has died out or was in the process of doing so. However, it should be recognized that in those areas the disappearance of leprosy was related more to improved social and economic conditions than to specific anti-leprosy measures. All the same, there is no reason to believe that resurgence is likely to be a serious factor as in the case of, say, malaria.

The next implication of the elimination strategy is to have a clear understanding of the level at which elimination should be attained. Is it global, regional, national, or subnational? This problem is quite important as the disease is extremely unevenly distributed among and within countries. Although the World Health Assembly resolution refers to global elimination, the inference is that such elimination should occur fairly evenly.

Another major implication of the elimination strategy is whether MDT will continue to be effective in the coming years. The elimination strategy is built around the efficacy of MDT together, of course, with case-finding. The experience with MDT over the past 6 to 8 years has given sufficient confidence in this direction. However, it is possible to foresee problems in the future with regard to treatment failure as a result either of inadequate treatment, improper application of MDT drugs leading to drug resistance or late relapses due to persisters. The indications are that the impact of such problems in leprosy control are likely to be very limited. All the same, research progress in the development of new drugs and improved MDT is sufficiently advanced so that it is quite conceivable that better MDT regimens, both from the points of view of efficacy as well as operational applicability, will become available towards the latter part of the 1990s.

The last and most important implication is whether elimination is feasible. In this connection, a point often raised is what is the basis for identifying a prevalence level of 1 in 10,000 as the required limit to be attained? The limit is clearly arbitrary but there is considerable hope and confidence that when such low levels of prevalence are reached the disease burden in the community, as well as the potential for transmission, will be extremely limited. The elimination resolution of the World Health Assembly is essentially a commitment of the Member States, with clear technical and political implications, and the goal is attainable provided leprosy control activities are intensified significantly enough so that coverage of MDT, together with case-finding, reaches the highest possible levels by the mid-1990s. Whether such intensification will be attained or not remains to be seen. Irrespective of political commitment and availability of resources, the situation is likely to be relatively more difficult in countries starting with a larger base of prevalence than in countries starting with a smaller base, warranting even more vigorous efforts.

Even as we are discussing and working actively towards our goal of elimination, we are likely to face several problems towards the latter phase of elimination. For example: difficult-to-reach cases, chronic defaulters, and possibly some relapses, all occurring within a background of a very low endemicity of around 1 in 10,000 population or less. At this time, we will require additional tools and additional strategies to deal with the remaining problems.

In conclusion, it is clear that there is still an immense amount of work that needs to be done if the target of elimination of leprosy as a public health problem is to be achieved by the year 2000, as envisaged by the World Health Assembly in its resolution adopted in May 1991. The target can be achieved provided that further, substantial, intensified 
efforts are made in terms both of action and mobilization of adequate resources. Such intensification is important, particularly during the next few years. For the leprosyendemic countries, it is an important opportunity that cannot be missed. It remains to be seen how this opportunity is exploited and thus a major public health problem solved. 